\section{Personal income, expenditure and wealth}

\section{Distribution of total incomes}

\section{(Table 8.1)}

The information shown in Table 8.1 comes from the Survey of Personal Incomes for the financial years 2003/04, 2004/05, $2005 / 06$ and $2006 / 07$. This is an annual survey that covers approximately 540,000 individuals across the whole of the UK. It is based on administrative data held by HM Revenue and Customs (HMRC) offices on individuals who could be liable to tax.

The table relates only to those individuals who are taxpayers. The distributions cover only incomes as computed for tax purposes and above a level which for each year corresponds approximately to the single person's allowance. Incomes below these levels are not shown because the information about them is incomplete.

Investment income from which tax has been deducted at source is not always known to local tax offices. Estimates of missing bank and building society interest and dividends from UK companies are included in these tables. The missing investment income is distributed, in a manner consistent with information from the Expenditure and Food Survey and the National Accounts, to individuals for whom there is no investment income already reported by the tax office.

Superannuation contributions are estimated and included in total income. They have been distributed among earners in the Survey of Personal Incomes sample by a method consistent with information about the number of employees who are contracted in or out of the State Earnings Related Pension Scheme and the proportion of their earnings contributed.

When comparing results of these surveys across years, it should be noted that the Survey of Personal Incomes is not a longitudinal survey. However, sample sizes have increased in recent years to increase precision.

\section{Average incomes of households}

\section{(Table 8.2)}

Original income is the total income in cash of all the members of the household before receipt of state benefits or the deduction of taxes. It includes income from employment, selfemployment, investment income and occupational pensions. Gross income is original income plus cash benefits received from government (retirement pensions, child benefit, etc). Disposal income is the income available for consumption. It is equal to gross income less direct taxes which include income tax, national insurance contributions, and council tax. By further allowing for taxes paid on goods and services purchased, such as VAT, an estimate of post-tax income is derived. These income figures are derived from estimates made by the Office for National Statistics, based largely on information from the Expenditure and Food Survey (EFS), and published each on the Office for National Statistics website.

For the purposes of Table 8.2, a retired household is defined as one where the combined income of retired members amounts to at least half the total gross income of the household, where a retired person is defined as anyone who describes themselves as 'retired' or anyone over the minimum $\mathrm{NI}$ pension age describing themselves as 'unoccupied' or 'sick or injured but not intending to seek work'.

Children are defined as persons aged under 16 or aged between 16 and 18, unmarried and receiving full-time nonadvanced further education.

\section{Expenditure and Food Survey}

\section{(Tables 8.3 to 8.5 )}

The Expenditure and Food Survey (EFS) is a sample survey of 11,484 private households in the UK, with an achieved response of around 6,200 private households. The survey was introduced in April 2001 as a result of the amalgamation of the Family Expenditure and National Food Surveys (FES and NFS). The EFS sample is representative of all regions of the UK and of different types of households. The survey is continuous with interviews spread evenly over the year to ensure that estimates are not biased by seasonal variation. The survey results show how households spend their money - how much goes on food, clothing and so on - and how spending patterns vary depending upon income, household composition, and regional location of households. From January 2006, the survey has been conducted on a calendar year basis; therefore the latest results refer to the January to December 2007 period.

One of the main purposes of the EFS is to define the 'basket of goods' for the Retail Price Index (RPI) and the Consumer Price Index (CPI). The RPI has a vital role in the up rating of state pensions and welfare benefits, while the CPI is a key instrument of the Government's monetary policy. Information from the survey is also a major source for estimates of household expenditure in the UK National Accounts. In 
Personal income, expenditure and wealth

addition, many other government departments use EFS data as a basis for policy making, for example in the areas of housing and transport. The Department for Environment, Food and Rural Affairs (Defra) uses EFS data to report on trends in food consumption and nutrient intake within the UK. Users of the EFS outside government include independent research institutes, academic researcher and business and market researchers. Like all surveys based on a sample of the population, its results are subject to sampling variability and potentially to some bias due to non-response. The results of the survey are published in an annual report, the latest being Family Spending 2008 edition. The report includes a list of definitions used in the survey, items on which information is collected and a brief account of the fieldwork procedure. 
Personal income, expenditure and wealth

\section{Q Distribution of total income before and after tax United Kingdom \\ Years ending 5 April}

\begin{tabular}{|c|c|c|c|c|c|c|c|c|c|}
\hline & \multicolumn{4}{|c|}{ 2003/2004 Annual Survey } & & \multicolumn{4}{|c|}{ 2004/05 Annual Survey } \\
\hline & \multirow{2}{*}{$\begin{array}{r}\text { Number of } \\
\text { individuals } \\
\text { (Thousands) }\end{array}$} & \multicolumn{3}{|c|}{$£$ million } & & \multirow{2}{*}{$\begin{array}{r}\text { Number of } \\
\text { individuals } \\
\text { (Thousands) }\end{array}$} & \multicolumn{3}{|c|}{$£$ million } \\
\hline & & $\begin{array}{l}\text { Total income } \\
\text { before tax }\end{array}$ & Total tax & $\begin{array}{r}\text { Total income } \\
\text { after tax }\end{array}$ & & & $\begin{array}{r}\text { Total income } \\
\text { before tax }\end{array}$ & Total tax & $\begin{array}{r}\text { Total income } \\
\text { after tax }\end{array}$ \\
\hline $\begin{array}{l}\text { Lower limit of } \\
\text { range of income }\end{array}$ & & & & & $\begin{array}{l}\text { Lower limit of } \\
\text { range of income }\end{array}$ & & & & \\
\hline All incomes ${ }^{1}$ & 28500 & 624900 & 110600 & 514300 & All incomes ${ }^{1}$ & 30300 & 691000 & 123000 & 568000 \\
\hline $\begin{array}{l}\text { Income before tax } \\
\text { (£) }\end{array}$ & & & & & $\begin{array}{r}\text { Income before tax } \\
(£)\end{array}$ & & & & \\
\hline 4615 & 498 & 2390 & 8 & 2380 & 4745 & 329 & 1600 & 4 & 1600 \\
\hline 5000 & 1090 & 6000 & 93 & 5900 & 5000 & 1110 & 6090 & 80 & 6010 \\
\hline 6000 & 2710 & 19100 & 636 & 18400 & 6000 & 2760 & 19500 & 600 & 18900 \\
\hline 8000 & 2660 & 23900 & 1570 & 22300 & 8000 & 2950 & 26500 & 1600 & 24900 \\
\hline 10000 & 2570 & 28300 & 2500 & 25800 & 10000 & 2760 & 30300 & 2580 & 27700 \\
\hline 12000 & 2430 & 31600 & 3400 & 28200 & 12000 & 2470 & 32100 & 3350 & 28700 \\
\hline 14000 & 2270 & 33900 & 4130 & 29800 & 14000 & 2280 & 34200 & 4080 & 30100 \\
\hline 16000 & 1990 & 33700 & 4450 & 29300 & 16000 & 2050 & 34800 & 4520 & 30300 \\
\hline 18000 & 1730 & 32900 & 4610 & 28300 & 18000 & 1790 & 34100 & 4720 & 29300 \\
\hline 20000 & 5710 & 139400 & 21800 & 117500 & 20000 & 6000 & 146000 & 22700 & 124000 \\
\hline 30000 & 3360 & 124500 & 22800 & 101700 & 30000 & 4090 & 152000 & 27300 & 125000 \\
\hline 50000 & 1110 & 73600 & 19300 & 54200 & 50000 & 1270 & 83700 & 21600 & 62100 \\
\hline 100000 & 256 & 34000 & 10800 & 23200 & 100000 & 300 & 40000 & 12600 & 27400 \\
\hline 200000 and over & 95 & 41600 & 14400 & 27200 & 200000 and over & 111 & 49500 & 17300 & 32200 \\
\hline $\begin{array}{l}\text { Income after tax } \\
(£)\end{array}$ & & & & & $\begin{array}{r}\text { Income after tax } \\
(£)\end{array}$ & & & & \\
\hline 4615 & 545 & 2620 & 10 & 2610 & 4745 & 364 & 1770 & 5 & 1770 \\
\hline 5000 & 1220 & 6820 & 116 & 6710 & 5000 & 1220 & 6830 & 98 & 6730 \\
\hline 6000 & 3190 & 23500 & 955 & 22500 & 6000 & 3270 & 24100 & 902 & 23200 \\
\hline 8000 & 3270 & 31900 & 2470 & 29400 & 8000 & 3600 & 34800 & 2510 & 32300 \\
\hline 10000 & 3160 & 38600 & 3920 & 34700 & 10000 & 3280 & 40000 & 3920 & 36000 \\
\hline 12000 & 2890 & 42600 & 5120 & 37500 & 12000 & 2920 & 43000 & 5050 & 37900 \\
\hline 14000 & 2400 & 42400 & 5660 & 36800 & 14000 & 2540 & 43700 & 5730 & 38000 \\
\hline 16000 & 2090 & 41300 & 5910 & 35400 & 16000 & 2180 & 43200 & 6090 & 37100 \\
\hline 18000 & 1720 & 38400 & 5840 & 32600 & 18000 & 1850 & 41400 & 6210 & 35200 \\
\hline 20000 & 4900 & 141600 & 23300 & 118300 & 20000 & 5320 & 154000 & 25100 & 129000 \\
\hline 30000 & 2280 & 106000 & 22800 & 83200 & 30000 & 2840 & 131000 & 27100 & 104000 \\
\hline 50000 & 601 & 55700 & 16300 & 39400 & 50000 & 681 & 63200 & 18300 & 44800 \\
\hline 100000 & 119 & 23800 & 7930 & 15800 & 100000 & 143 & 28400 & 9420 & 19000 \\
\hline 200000 and over & 45 & 29600 & 10200 & 19300 & 200000 and over & 53 & 35500 & 12500 & 23000 \\
\hline
\end{tabular}


Personal income, expenditure and wealth

8.1

Distribution of total income before and after tax

United Kingdom

continued Years ending 5 April

\begin{tabular}{|c|c|c|c|c|c|c|c|c|c|}
\hline & \multicolumn{4}{|c|}{ 2005/06 Annual Survey } & & \multicolumn{4}{|c|}{ 2006/07 Annual Survey } \\
\hline & \multirow{2}{*}{$\begin{array}{c}\text { Number of } \\
\text { individuals } \\
\text { (Thousands) }\end{array}$} & \multicolumn{3}{|c|}{$£$ million } & & \multirow{2}{*}{$\begin{array}{r}\text { Number of } \\
\text { individuals } \\
\text { (Thousands) }\end{array}$} & \multicolumn{3}{|c|}{$£$ million } \\
\hline & & $\begin{array}{r}\text { Total income } \\
\text { before tax }\end{array}$ & Total tax & $\begin{array}{r}\text { Total income } \\
\text { after tax }\end{array}$ & & & $\begin{array}{r}\text { Total income } \\
\text { before tax }\end{array}$ & Total tax & $\begin{array}{r}\text { Total income } \\
\text { after tax }\end{array}$ \\
\hline $\begin{array}{l}\text { Lower limit of } \\
\text { range of income }\end{array}$ & & & & & $\begin{array}{l}\text { Lower limit of } \\
\text { range of income }\end{array}$ & & & & \\
\hline All incomes ${ }^{1}$ & 31100 & 756000 & 138000 & 618000 & All incomes ${ }^{1}$ & 31800 & 810000 & 150000 & 661000 \\
\hline $\begin{array}{l}\text { Income before tax } \\
(£)\end{array}$ & & & & & $\begin{array}{r}\text { Income before tax } \\
(£)\end{array}$ & & & & \\
\hline 4895 & 112 & 555 & - & 555 & - & - & - & - & \\
\hline 5000 & 1040 & 5750 & 62 & 5690 & 5035 & 919 & 5090 & 43 & 5050 \\
\hline 6000 & 2540 & 18000 & 522 & 17500 & 6000 & 2440 & 17200 & 451 & 16800 \\
\hline 8000 & 2920 & 26200 & 1450 & 24800 & 8000 & 2920 & 26200 & 1330 & 24900 \\
\hline 10000 & 2810 & 30900 & 2500 & 28400 & 10000 & 2790 & 30600 & 2390 & 28200 \\
\hline 12000 & 2550 & 33100 & 3380 & 29700 & 12000 & 2570 & 33400 & 3310 & 30100 \\
\hline 14000 & 2340 & 35000 & 4140 & 30900 & 14000 & 2400 & 36000 & 4180 & 31800 \\
\hline 16000 & 2100 & 35700 & 4610 & 31100 & 16000 & 2140 & 36300 & 4630 & 31700 \\
\hline 18000 & 1880 & 35700 & 4930 & 30800 & 18000 & 1970 & 37300 & 5110 & 32200 \\
\hline 20000 & 6200 & 152000 & 23400 & 128000 & 20000 & 6530 & 160000 & 24600 & 135000 \\
\hline 30000 & 4540 & 170000 & 29900 & 140000 & 30000 & 4900 & 184000 & 32000 & 152000 \\
\hline 50000 & 1500 & 98800 & 25000 & 73700 & 50000 & 1670 & 110000 & 27400 & 82600 \\
\hline 100000 & 366 & 49300 & 15300 & 34000 & 100000 & 406 & 54700 & 16700 & 38000 \\
\hline 200000 and over & 144 & 66000 & 22900 & 43000 & 200000 and over & 170 & 79700 & 27400 & 52300 \\
\hline $\begin{array}{l}\text { Income after tax } \\
(£)\end{array}$ & & & & & $\begin{array}{r}\text { Income after tax } \\
(£)\end{array}$ & & & & \\
\hline 4895 & 129 & 636 & 1 & 636 & - & - & - & - & - \\
\hline 5000 & 1160 & 6500 & 77 & 6420 & 5035 & 1040 & 5800 & 55 & 5750 \\
\hline 6000 & 3000 & 22000 & 767 & 21300 & 6000 & 2860 & 20900 & 656 & 20200 \\
\hline 8000 & 3590 & 34600 & 2300 & 32300 & 8000 & 3550 & 34000 & 2090 & 31900 \\
\hline 16000 & 2260 & 44600 & 6270 & 38300 & 16000 & 2370 & 46800 & 6530 & 40200 \\
\hline 18000 & 1850 & 41300 & 6140 & 35100 & 18000 & 1920 & 42700 & 6320 & 36400 \\
\hline 20000 & 5630 & 163000 & 26400 & 137000 & 20000 & 5980 & 173000 & 28000 & 145000 \\
\hline 30000 & 3310 & 152000 & 30700 & 121000 & 30000 & 3650 & 168000 & 33200 & 134000 \\
\hline 50000 & 817 & 75500 & 21600 & 53900 & 50000 & 914 & 83800 & 23600 & 60200 \\
\hline 100000 & 188 & 37100 & 12100 & 24900 & 100000 & 220 & 43300 & 13900 & 29400 \\
\hline 200000 and over & 69 & 47900 & 16800 & 31100 & 200000 and over & 82 & 58600 & 20200 & 38300 \\
\hline
\end{tabular}

1 See chapter text. All figures have been independently rounded.

Sources: Survey of Personal Incomes; Board of HM Revenue \& Customs:020 74387055 


\begin{tabular}{|c|c|c|c|c|c|c|c|c|c|c|c|}
\hline & \multicolumn{2}{|c|}{ Retired households } & \multicolumn{9}{|c|}{ Non-retired households } \\
\hline & 1 adult & $\begin{array}{r}2 \text { or } \\
\text { more } \\
\text { adults }\end{array}$ & 1 adult & 2 adults & $\begin{array}{r}3 \text { or } \\
\text { more } \\
\text { adults }\end{array}$ & $\begin{array}{r}1 \text { adult } \\
\text { with } \\
\text { children }\end{array}$ & $\begin{array}{r}2 \text { adults } \\
\text { with } \\
1 \text { child }\end{array}$ & $\begin{array}{r}2 \text { adults } \\
\text { with } 2 \\
\text { children }\end{array}$ & $\begin{array}{r}2 \text { adults } \\
\text { with } 3 \\
\text { or more } \\
\text { children }\end{array}$ & $\begin{array}{r}3 \text { or } \\
\text { more } \\
\text { adults } \\
\text { with } \\
\text { children }\end{array}$ & $\begin{array}{r}\text { All } \\
\text { house- } \\
\text { holds }\end{array}$ \\
\hline $\begin{array}{l}\text { Number of households in } \\
\text { the population (thousands) }\end{array}$ & 3291 & 3234 & 3638 & 5260 & 2172 & 1441 & 1847 & 2176 & 812 & 965 & 24836 \\
\hline \multicolumn{12}{|l|}{$\begin{array}{l}\text { Average per household } \\
\text { ( } £ \text { per year) }\end{array}$} \\
\hline Original income & 5020 & 13047 & 22053 & 42649 & 48549 & 11698 & 43084 & 48056 & 41866 & 44947 & 30080 \\
\hline Gross income & 12387 & 22800 & 24153 & 44681 & 51476 & 19331 & 45778 & 51141 & 48062 & 50704 & 34661 \\
\hline Disposable income & 10970 & 19522 & 18545 & 34050 & 40410 & 16988 & 35412 & 39270 & 38272 & 41237 & 27370 \\
\hline Post-tax income & 8951 & 15400 & 15379 & 28081 & 32862 & 13526 & 29485 & 32464 & 31920 & 33193 & 22420 \\
\hline
\end{tabular}

1 See chapter text. Figures taken from the article "Effects of taxes and benefits on household income, 2006/07", published on the National Statistics website www.statistics.gov.uk/taxesbenefits

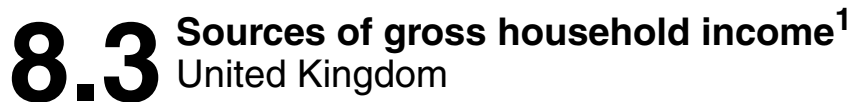

\begin{tabular}{|c|c|c|c|c|c|c|c|c|c|c|c|c|c|}
\hline & & $\begin{array}{r}1996 \\
/ 97\end{array}$ & $\begin{array}{r}1997 \\
/ 98\end{array}$ & $\begin{array}{c}1998^{2} \\
199\end{array}$ & $\begin{array}{r}1999 \\
100\end{array}$ & $\begin{array}{r}2000 \\
/ 01\end{array}$ & $\begin{array}{c}2001^{3} \\
/ 02\end{array}$ & $\begin{array}{r}2002 \\
103\end{array}$ & $\begin{array}{r}2003 \\
104\end{array}$ & $\begin{array}{r}2004 \\
105\end{array}$ & $\begin{array}{r}2005 \\
106\end{array}$ & $2006^{4,5}$ & 52007 \\
\hline Weighted number of households (thousands) & GH92 & 24310 & 24560 & 24660 & 25330 & 25030 & 24450 & 24350 & 24670 & 24430 & 24800 & 25440 & 25350 \\
\hline Number of households supplying data & KPDA & 6420 & 6410 & 6630 & 7100 & 6640 & 7470 & 6930 & 7050 & 6800 & 6790 & 6650 & 6140 \\
\hline \multicolumn{14}{|l|}{$\begin{array}{l}\text { Average weekly household } \\
\text { income by source }(£)\end{array}$} \\
\hline Wages and salaries & $\mathrm{KPCB}$ & 256.30 & 280.20 & 309.20 & 315.40 & 336.70 & 369.30 & 373.90 & 383.90 & 409.70 & 414.80 & 428.20 & 444.90 \\
\hline Investments & $\mathrm{KPCD}$ & 17.70 & 18.70 & 18.80 & 21.80 & 20.00 & 20.00 & 18.80 & 16.70 & 16.50 & 19.50 & 20.90 & 23.20 \\
\hline Annuities and pensions (other & & & & & & & & & & & & & \\
\hline than social security benefits) & $\mathrm{KPCE}$ & 26.00 & 28.90 & 30.30 & 32.80 & 35.00 & 37.00 & 39.90 & 40.90 & 41.70 & 45.50 & 44.40 & 47.60 \\
\hline Social security benefits ${ }^{6}$ & KPCF & 54.10 & 55.00 & 55.80 & 58.00 & 60.10 & 64.50 & 68.50 & 72.50 & 76.90 & 78.00 & 79.40 & 83.30 \\
\hline Other sources & $\mathrm{KPCH}$ & 5.30 & 5.20 & 5.70 & 5.90 & 6.20 & 6.70 & 6.70 & 6.40 & 6.90 & 7.40 & 6.50 & 7.00 \\
\hline Total $^{7}$ & KPCI & $\overline{396.90}$ & $\overline{420.80}$ & 457.00 & $\overline{479.90}$ & $\overline{502.50}$ & 540.60 & 552.30 & 570.30 & 600.70 & 615.90 & 634.70 & 659.40 \\
\hline
\end{tabular}

Sources of household income as a percentage of total household income

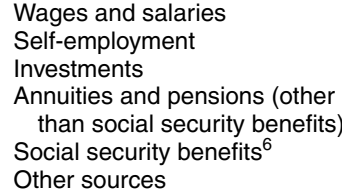

\begin{tabular}{|c|c|c|c|c|c|c|c|c|c|c|c|c|}
\hline KPCJ & 65 & 67 & 68 & 66 & 67 & 68 & 68 & 67 & 68 & 67 & 67 & 67 \\
\hline KPCK & 9 & 8 & 8 & 10 & 9 & 8 & 8 & 9 & 8 & 8 & 9 & 8 \\
\hline KPCL & 4 & 4 & 4 & 5 & 4 & 4 & 3 & 3 & 3 & 3 & 3 & 4 \\
\hline KPCM & 7 & 7 & 7 & 7 & 7 & 7 & 7 & 7 & 7 & 7 & 7 & 7 \\
\hline KPCN & 14 & 13 & 12 & 12 & 12 & 12 & 12 & 13 & 13 & 13 & 13 & 13 \\
\hline KPCP & 1 & 1 & 1 & 1 & 1 & 1 & 1 & 1 & 1 & 1 & 1 & 1 \\
\hline KPCQ & 100 & 100 & 100 & 100 & 100 & 100 & 100 & 100 & 100 & 100 & 100 & 100 \\
\hline
\end{tabular}

1 See chapter text

2 Based on weighted data from 1998/99.

3 From 2001/02 onwards, weighting is based on the population estimates from the 2001 census.

4 From 2006 the survey has moved onto a calendar year basis.

5 From 1998/99 to 2005/06, the figures shown are based on weighted data using non-response weights based on the 1991 Census and population figures from the 1991 and 2001 Census. From 2006, figures shown on weighted data using updated weigths, with non-response weights and population figures based on the 2001 Census.

6 Excluding housing benefit and council tax benefit (rates rebate in Northern Ireland) and their predecessors in earlier years.

7 Does not include imputed income from owner-occupied and rent-free occupancy.

Sources: Expenditure and Food Survey and Family Expenditure Survey; Office for National Statistics; 01633455282 


\begin{tabular}{|c|c|c|c|c|c|c|c|c|c|c|c|c|c|}
\hline & & $\begin{array}{r}1996 \\
/ 97\end{array}$ & $\begin{array}{r}1997 \\
/ 98\end{array}$ & $\begin{array}{c}1998^{2} \\
/ 99\end{array}$ & $\begin{array}{r}1999 \\
100\end{array}$ & $\begin{array}{r}2000 \\
/ 01\end{array}$ & $\begin{array}{c}2001^{3} \\
/ 02\end{array}$ & $\begin{array}{r}2002 \\
/ 03\end{array}$ & $\begin{array}{r}2003 \\
/ 04\end{array}$ & $\begin{array}{r}2004 \\
/ 05\end{array}$ & $\begin{array}{r}2005 \\
/ 06\end{array}$ & $2006^{4,5}$ & 2007 \\
\hline Weighted number of households (the & GH92 & 24310 & 24560 & 24660 & 25330 & 25030 & 24450 & 24350 & 24670 & 24430 & 24800 & 25440 & 25350 \\
\hline Number of households supplying data & KPDA & 6420 & 6410 & 6630 & 7100 & 6640 & 7470 & 6930 & 7050 & 6800 & 6790 & 6650 & 6140 \\
\hline \multicolumn{14}{|c|}{ Average weekly household expenditure on commodities and services ( $(\varepsilon)$} \\
\hline Housing $(\mathrm{NET})^{6}$ & KPEV & 49.10 & 51.50 & 57.20 & 57.00 & 63.90 & 65.90 & 66.70 & 69.90 & 76.70 & 80.90 & 83.20 & 92.00 \\
\hline Fuel and power & KPEW & 13.40 & 12.70 & 11.70 & 11.30 & 11.90 & 11.70 & 11.70 & 12.00 & 12.50 & 13.90 & 15.80 & 17.20 \\
\hline Alcoholic drink & KPEY & 12.40 & 13.30 & 14.00 & 15.30 & 15.00 & 14.30 & 14.80 & 14.70 & 14.80 & 14.80 & 14.70 & 14.70 \\
\hline Tobacco & KPEZ & 6.10 & 6.10 & 5.80 & 6.00 & 6.10 & 5.50 & 5.40 & 5.50 & 5.00 & 4.50 & 4.70 & 4.60 \\
\hline Clothing and footwear & KCWC & 18.30 & 20.00 & 21.70 & 21.00 & 22.00 & 22.30 & 22.00 & 22.40 & 23.50 & 22.40 & 22.60 & 21.60 \\
\hline Household goods & KCWH & 26.70 & 26.90 & 29.60 & 30.70 & 32.60 & 33.00 & 33.80 & 35.10 & 35.60 & 33.50 & 34.00 & 34.60 \\
\hline old service & KCWI & 16.40 & 17.90 & 18.90 & 18.90 & 22.00 & 23.60 & 23.30 & 24.90 & 26.30 & 27.10 & 26.40 & 26.50 \\
\hline Personal goods and services & KCWJ & 11.60 & 12.50 & 13.30 & 13.90 & 14.70 & 14.90 & 15.20 & 16.20 & 16.00 & 16.90 & 17.50 & 17.80 \\
\hline Motoring & KCWK & 41.20 & 46.60 & 51.70 & 52.60 & 55.10 & 57.90 & 61.70 & 62.40 & 62.60 & 63.80 & 61.10 & 62.00 \\
\hline Fares and other travel costs & KCWL & 7.50 & 8.10 & 8.30 & 9.20 & 9.50 & 9.30 & 9.70 & 9.60 & 9.50 & 11.10 & 11.00 & 10.90 \\
\hline Leisure goods & KCWM & 15.20 & 16.40 & 17.80 & 18.50 & 19.70 & 19.60 & 20.50 & 21.40 & 21.40 & 19.40 & 19.40 & 20.10 \\
\hline Total & KCWP & 09.10 & 8.80 & 2.20 & 9.40 & 85.70 & 3.90 & 4.70 & 15.70 & 32.90 & 41.40 & 47.40 & 56.80 \\
\hline
\end{tabular}

Expenditure on commodity or service as a percentage of total expenditure

\begin{tabular}{|c|c|c|c|c|c|c|c|c|c|c|c|c|c|}
\hline Housing $(\mathrm{NET})^{6}$ & KPFH & 16 & 16 & 16 & 16 & 17 & 17 & 16 & 17 & 18 & 18 & 19 & 20 \\
\hline Fuel and power & KPFI & 4 & 4 & 3 & 3 & 3 & 3 & 3 & 3 & 3 & 3 & 4 & 4 \\
\hline Food and non-alcoholic drinks & KPFJ & 18 & 17 & 17 & 17 & 16 & 16 & 16 & 16 & 16 & 15 & 16 & 16 \\
\hline Alcoholic drink & KPFK & 4 & 4 & 4 & 4 & 4 & 4 & 4 & 4 & 3 & 3 & 3 & 3 \\
\hline Tobacco & KPFL & 2 & 2 & 2 & 2 & 2 & 1 & 1 & 1 & 1 & 1 & 1 & 1 \\
\hline Household goods & KCWQ & 9 & 8 & 8 & 9 & 8 & 8 & 8 & 8 & 8 & 8 & 8 & 8 \\
\hline Household services & KCWR & 5 & 5 & 5 & 5 & 6 & 6 & 6 & 6 & 6 & 6 & 6 & 6 \\
\hline Personal goods and services & KCWS & 4 & 4 & 4 & 4 & 4 & 4 & 4 & 4 & 4 & 4 & 4 & 4 \\
\hline Motoring & KCWT & 13 & 14 & 15 & 15 & 14 & 15 & 15 & 15 & 14 & 14 & 14 & 14 \\
\hline Fares and other travel costs & KCWU & 2 & 2 & 2 & 3 & 2 & 2 & 2 & 2 & 2 & 3 & 2 & 2 \\
\hline Leisure goods & KCWV & 5 & 5 & 5 & 5 & 5 & 5 & 5 & 5 & 5 & 4 & 4 & 4 \\
\hline Leisure services & KCWW & 11 & 12 & 12 & 12 & 13 & 13 & 13 & 13 & 14 & 14 & 15 & 13 \\
\hline Miscellaneous & KPFR & 1 & 1 & - & - & - & - & - & - & - & - & - & - \\
\hline otal & KPFS & 100 & 100 & 100 & 100 & 100 & 100 & 100 & 100 & 100 & 100 & 100 & 100 \\
\hline
\end{tabular}

1 Data are based on the Family Expenditure Survey (FES) classification and 4 From 2006 the survey has moved onto a calendar year basis.

not the Expenditure and Food Survey (EFS) standard classification: Classi- 5 From 1998/99 to 2005/06, the figures shown are based on weighted data using fication of Individual Consumption by Purpose (COICOP). This has been done to preserve an historical time-series, as COICOP data are only available from 2001/02.

2 From 1998/99 figures shown are based on weighted data, including children's expenditure. Weighting is based on the population figures from the 1991 and 2001 Censuses.

3 From 2001/02 onwards, weighting is based on population estimates from the 2001 census. non-response weights based on the 1991 Census and population figures from the 1991 and 2001 Census. From 2006, figures shown are based on weighted data using updated weights, with non-response weights and population figures based on the 2001 Census.

6 An improvement to the implation of mortgage intrest payments has been implemented for 2006 and 2007 data which should lead to more accurate figures. This will lead to a slight discontinuity. An error was discovered in the derivation of mortgage capital repayments which was leading to double counting. This has been amended for the 2006 and 2007 data.

Sources: Expenditure and Food Survey and Family Expenditure Survey; Office for National Statistics 01633455282 


\begin{tabular}{|c|c|c|c|c|c|c|c|c|c|c|c|c|c|}
\hline & & $\begin{array}{r}1996 \\
/ 97\end{array}$ & $\begin{array}{r}1997 \\
/ 98\end{array}$ & $\begin{array}{c}1998^{1} \\
/ 99\end{array}$ & $\begin{array}{r}1999 \\
100\end{array}$ & $\begin{array}{r}2000 \\
/ 01\end{array}$ & $\begin{array}{c}2001^{2} \\
/ 02\end{array}$ & $\begin{array}{r}2002 \\
/ 03\end{array}$ & $\begin{array}{r}2003 \\
104\end{array}$ & $\begin{array}{r}2004 \\
105\end{array}$ & $\begin{array}{r}2005 \\
106\end{array}$ & $2006^{4,5}$ & 52007 \\
\hline Weighted number of households (thousands) & GH92 & 24310 & 24560 & 24660 & 25330 & 25030 & 24450 & 24350 & 24670 & 24430 & 24800 & 254402 & 25350 \\
\hline Number of households supplying data & KPDA & 6420 & 6410 & 6630 & 7100 & 6640 & 7470 & 6930 & 7050 & 6800 & 6790 & 6650 & 6140 \\
\hline Car/van & $\mathrm{KPDB}$ & 69 & 70 & 72 & 71 & 72 & 74 & 74 & 75 & 75 & 74 & 74 & 75 \\
\hline One & KPDC & 43 & 44 & 44 & 43 & 44 & 44 & 44 & 44 & 42 & 46 & 43 & 44 \\
\hline Two & KPDD & 22 & 21 & 23 & 21 & 22 & 23 & 25 & 25 & 27 & 23 & 25 & 25 \\
\hline Three or more & KPDE & 5 & 5 & 5 & 6 & 6 & 6 & 6 & 6 & 6 & 5 & 6 & 6 \\
\hline \multicolumn{14}{|l|}{ Central heating, full or } \\
\hline partial & KPDF & 87 & 89 & 89 & 90 & 91 & 92 & 93 & 94 & 95 & 94 & 95 & 95 \\
\hline Washing machine & KPDG & 91 & 91 & 92 & 91 & 92 & 93 & 94 & 94 & 95 & 95 & 96 & 96 \\
\hline Tumble dryer & J803 & 51 & 51 & 51 & 52 & 53 & 54 & 56 & 57 & 58 & 58 & 59 & 57 \\
\hline \multicolumn{14}{|l|}{ Fridge/freezer } \\
\hline or deep freezer & KPDI & 91 & 90 & 92 & 91 & 94 & 95 & 96 & 96 & 96 & 97 & 97 & 97 \\
\hline Dishwasher & GPTL & 20 & 22 & 23 & 23 & 25 & 27 & 29 & 31 & 33 & 35 & 37 & 37 \\
\hline Microwave & J804 & 75 & 77 & 79 & 80 & 84 & 86 & 87 & 89 & 90 & 91 & 91 & 91 \\
\hline Telephone & KPDL & 93 & 94 & 95 & 95 & 93 & 94 & 94 & 92 & 93 & 92 & 91 & 89 \\
\hline Mobile phone & GH9 6 & 16 & 20 & 27 & 44 & 47 & 64 & 70 & 76 & 78 & 79 & 79 & 78 \\
\hline Home computer & KPDM & 27 & 29 & 33 & 38 & 44 & 49 & 55 & 58 & 62 & 65 & 67 & 70 \\
\hline Video recorder & KPDN & 82 & 84 & 85 & 86 & 87 & 90 & 90 & 90 & 88 & 86 & 82 & 75 \\
\hline DVD player & J805 & .. & .. &.. & .. & .. & .. & 31 & 50 & 67 & 79 & 83 & 86 \\
\hline CD player & J806 & 59 & 63 & 68 & 72 & 77 & 80 & 83 & 86 & 87 & 88 & 87 & 86 \\
\hline Digital television service ${ }^{3}$ & GH9 7 & 19 & 26 & 28 & 32 & 40 & 43 & 45 & 49 & 58 & 65 & 70 & 77 \\
\hline Internet connection & ZBUZ & .. & .. & 10 & 19 & 32 & 39 & 45 & 49 & 53 & 55 & 58 & 61 \\
\hline
\end{tabular}

1 Based on weighted data from 1998/99

Sources: Expenditure and Food Survey and Family Expenditure Survey;

2 From 2001/02 onwards, weighting is based on the population estimates

Office for National Statistics; from the 2001 census.

01633455282

3 Includes digital, satellite and cable receivers.

4 From 2006 the survey has moved onto a calendar year basis.

5 From 1998/99 to 2005/06, the figures shown are based on weighted data using non-response weights based on the 1991 Census and population figures from the 1991 and 2001 Census. From 2006, figures shown are based on weighted data using updated weights, with non-response weights and population figures based on the 2001 Census. 